\title{
Serial Noninvasive Assessment of Apoptosis During Right Ventricular Disease Progression in Rats
}

Maria E. Campian ${ }^{1}$, Hein J. Verberne ${ }^{2}$, Maxim Hardziyenka ${ }^{1}$, Kora de Bruin ${ }^{2}$, Mariana Selwaness ${ }^{1}$, Maurice J.B. van den Hoff ${ }^{1}$, Jan M. Ruijter ${ }^{1}$, Berthe L.F. van Eck-Smit ${ }^{2}$, Jacques M.T. de Bakker ${ }^{1}$, and Hanno L. Tan ${ }^{1,3}$

${ }^{1}$ Heart Failure Research Center, Academic Medical Center, Amsterdam, The Netherlands; ${ }^{2}$ Department of Nuclear Medicine, Academic Medical Center, University of Amsterdam, Amsterdam, The Netherlands; and ${ }^{3}$ Department of Cardiology, Academic Medical Center, University of Amsterdam, Amsterdam, The Netherlands

Right ventricular (RV) function is the major determinant of survival in patients with pulmonary hypertension. Yet, the pathophysiologic basis of RV disease is unresolved. We aimed to study the role of apoptosis in RV disease by monitoring it serially during disease progression using in vivo 99mTc-annexin-V (99mTc-annexin) scintigraphy and study whether the reduction in apoptosis resulting from chronic treatment with valsartan can be detected by ${ }^{99 m}$ Tc-annexin scintigraphy. Methods: RV disease after pulmonary hypertension was induced by monocrotaline injection in rats. The following 3 groups were studied: rats treated with monocrotaline (monocrotaline rats), rats treated with monocrotaline plus valsartan (valsartan rats), and age-matched controls (control rats). Serial echocardiography and in vivo $99 \mathrm{~m} T c-a n n e x i n$ scintigraphy were performed. Apoptosis was confirmed by $99 \mathrm{mTc}$-annexin autoradiography and terminal deoxynucleotidyl-transferase-mediated dUTP nick-end labeling. Fibrosis was assessed by picrosirius red staining. Results: In monocrotaline rats, in vivo ${ }^{99 m} \mathrm{Tc}$-annexin uptake peaked early and declined thereafter but remained elevated, compared with baseline. These stage-dependent changes of in vivo 99mTc-annexin uptake were paralleled by changes in autoradiography and terminal deoxynucleotidyl-transferase-mediated dUTP nick-end labeling. Valsartan rats had longer RV failurefree survival than did monocrotaline rats and had reduced apoptosis. These changes were accompanied by commensurate delays in RV hypertrophy and RV dilation. Valsartan rats also had less fibrosis than monocrotaline rats at all disease stages. Conclusion: RV disease progression is associated with an early increase in RV apoptosis, as monitored using serial in vivo ${ }^{99 m}$ Tc-annexin scintigraphy. Delay in RV disease progression by valsartan is accompanied by reduction in $\mathrm{RV}$ apoptosis. Apoptosis plays a role in RV disease progression and may be assessed by serial in vivo ${ }^{99 m}$ Tc-annexin scintigraphy.

Key Words: right ventricle; heart failure; hypertrophy; apoptosis; fibrosis; annexin

J Nucl Med 2009; 50:1371-1377

DOI: 10.2967/jnumed.108.061366

Received Dec. 19, 2008; revision accepted Apr. 13, 2009.

For correspondence or reprints contact: Hanno L. Tan, Heart Failure Research Center, Academic Medical Center, Meibergdreef 91105 AZ, Amsterdam, The Netherlands.

E-mail: h.l.tan@amc.nl

COPYRIGHT $\odot 2009$ by the Society of Nuclear Medicine, Inc.
$\mathbf{R}$ ventricular (RV) disease receives increasing clinical recognition (1). $\mathrm{RV}$ dysfunction is the major determinant of survival in patients with pulmonary hypertension, regardless of the underlying cause (2). However, the pathophysiologic basis of RV disease is unresolved (3).

The molecular mechanisms that underlie left ventricular (LV) disease progression have been intensely studied (4-6). It is generally accepted that apoptosis (7) plays an important role in LV disease. Moreover, accumulation of collagen was demonstrated in hypertrophied and failing hearts (8). Apoptosis and fibrosis have been shown to act in isolation or in concert to reduce LV performance $(9,10)$. In contrast, the association of apoptosis with RV disease progression $(11,12)$ is unclear. Recently, in vivo imaging of cardiac apoptosis with ${ }^{99 m}$ Tc-annexin $\mathrm{V}$ ( ${ }^{99 m}$ Tc-annexin) was proven feasible, because ${ }^{99 \mathrm{~m}} \mathrm{Tc}$-annexin binds to exposed phosphatidylserine on the outer surface of apoptotic cells (13). These studies demonstrated apoptosis at single time points. Clearly, serial monitoring of apoptosis in vivo is relevant. This not only may provide insight into the time course of apoptosis but also may aid in determining the optimal timing of antiapoptotic therapy and in assessing the efficacy of therapy. We aimed to study the role of apoptosis in RV disease progression with serial in vivo ${ }^{99 \mathrm{~m}} \mathrm{Tc}$-annexin scintigraphy. Furthermore, we aimed to establish whether reduction in apoptosis resulting from treatment with the angiotensin II receptor-1 blocker (ARB) valsartan can be detected in vivo. ARBs have been shown to exert beneficial effects on RV disease (14). These effects may be due to the inhibition of apoptosis, as angiotensin II is a mediator of apoptosis $(15,16)$.

\section{MATERIALS AND METHODS}

\section{Study Design}

This investigation was performed in accordance with the Guide for the Care and Use of Laboratory Animals (17). Male 8-wk-old Wistar rats, weighing $225-285 \mathrm{~g}$ at the beginning of the experiment, were studied. RV disease was induced with a single 
intraperitoneal injection ( $60 \mathrm{mg} / \mathrm{kg}$ of body weight) of monocrotaline. Monocrotaline injection leads to severe pulmonary disease in the absence of intrinsic heart and lung disease (18). The ensuing pulmonary hypertension results in RV hypertrophy and RV failure. We monitored changes in RV function and apoptosis over time in untreated rats (monocrotaline rats) and rats that received longterm treatment with valsartan $(10 \mathrm{mg} / \mathrm{kg} / \mathrm{d}$, dissolved in saline, kindly provided by Novartis), administered via osmotic minipumps (Alzet) that were implanted subcutaneously directly before monocrotaline injection (valsartan rats). As a control group (control rats), we used age-matched rats that received intraperitoneal saline with or without valsartan via osmotic minipumps; we pooled these groups because there were no significant differences between them.

Cardiac function and in vivo apoptosis were serially monitored by echocardiography and in vivo ${ }^{99 \mathrm{~m}} \mathrm{Tc}$-annexin scintigraphy, respectively, in 10 rats of each treatment group (control, monocrotaline, and valsartan). Measurements were performed twice a week, and the rats were sacrificed after a maximum of $6 \mathrm{wk}$ or when RV failure occurred. To distinguish whether the in vivo ${ }^{99 \mathrm{~m}} \mathrm{Tc}$-annexin signal originated in the right or left ventricle, we harvested hearts at each of 3 stages of RV disease progression (RV hypertrophy $[n=3]$, RV dilation $[n=3]$, and RV failure $[n=4]$ ) and performed ex vivo tracer autoradiography in 10 control rats and 10 monocrotaline rats. In addition, 12 control rats, 12 monocrotaline rats, and 12 valsartan rats were sacrificed for terminal deoxynucleotidyl-transferase-mediated dUTP nick-end labeling (TUNEL) assays ( $n=4$ at each RV disease stage). RV hypertrophy and RV dilation were identified using serial echocardiography. RV failure was defined by clinical signs (body weight loss, cyanotic ears, cold limbs, and dyspnea) (19).

\section{Echocardiography}

Transthoracic 2-dimensional, M-mode and Doppler echocardiography was performed in accordance with the standards of the American Society of Echocardiography, using a 10-MHz transducer in anesthetized, spontaneously breathing rats (3\% isoflurane). The dose of the anesthetic was reduced by $50 \%$ in RV-failure rats. RV free wall thickness (RVFWT) was measured in the 2-dimensional short-axis parasternal view below the tricuspid valve or in the long-axis parasternal view by M-mode, depending on the quality of visualization. RV end-diastolic diameter (RVEDD) was measured as the maximal distance from the RV free wall to the interventricular septum from the apical 4-chamber view. Each parameter was averaged over 3 cardiac cycles. RV hypertrophy was defined by an RVFWT greater than $0.7 \mathrm{~mm}(22.2 \pm 1.7 \mathrm{~d}$ after monocrotaline injection in monocrotaline rats) and RV dilation by an RVEDD greater than $4.5 \mathrm{~mm}(27.0 \pm 1.7 \mathrm{~d}$ in monocrotaline rats) (19).

\section{9mTc-Annexin Scintigraphy}

After the echocardiographic recordings, ${ }^{99 m}$ Tc-annexin (40 $\mathrm{MBq}$ ) was injected into a tail vein. Anterior planar scintigraphy was performed $1 \mathrm{~h}$ thereafter, with a dedicated single-pinhole system designed for SPECT (20). Acquisition in the left anterior oblique position was not feasible because of overprojection of nonspecific bone marrow uptake. Rats were immobilized in an acrylic cylinder, mounted on a stepping-motor-driven system and positioned above the up-facing pinhole collimator of the $\gamma$-camera. This enabled anterior scintigraphy at a standardized orientation and distance from the pinhole aperture. In the present study, a tungsten insert with a 3-mm pinhole aperture was used.
SPECT images could not be reliably reconstructed because of minimal myocardial ${ }^{99 \mathrm{~m}} \mathrm{Tc}$-annexin uptake. SPECT is therefore not part of the current analysis. The $\gamma$-camera was connected to a HERMES acquisition and processing station. Static images of the thorax were obtained for $20 \mathrm{~min}$, with a $20 \%$ energy window at the $140-\mathrm{keV}{ }^{99 \mathrm{~m}} \mathrm{Tc}$ photon peak in a $128 \times 128$ matrix. One standardized region of interest was drawn over the myocardium (specific activity) and 2 over extrathoracic soft tissue in the axilla (nonspecific activity). As there were no differences in the results for the 2 axillary regions of interest, the data were combined to produce aggregate axillary uptake data for further analysis. The myocardial uptake ratio was calculated as the ratio of specific and nonspecific uptake (expressed as mean counts per pixel) as follows: (myocardium-nonspecific)/nonspecific. The image analyzer was unaware of the disease stage of the rats.

\section{Tracer Autoradiography}

After in vivo scintigraphy, the animals were sacrificed, and their hearts were used for tracer autoradiography with phosphor imaging. The hearts were excised, quickly frozen, and sliced into 50$\mu \mathrm{m}$ short-axis slices. Every fifth slice was mounted on a glass plate and covered with plastic wrap to prevent contamination of the phosphor plate. The short-axis slices were exposed to a Fuji BAS-MS imaging plate for approximately $24 \mathrm{~h}$. The images were scanned at a $50-\mu \mathrm{m}$ resolution with a 16-bit pixel depth using a Fuji FLA-3000 phosphor imager and analyzed using AIDA image

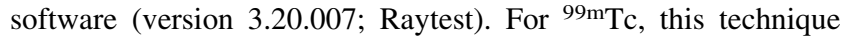
has a strong linear relationship between activity and photostimulated luminescence and a high resolution of $0.35 \pm 0.06$ $\mathrm{mm}$, expressed as full width at half maximum (21).

\section{TUNEL}

In situ TUNEL was performed using an apoptosis detection kit (In Situ Apoptosis Detection Kit POD; Roche) (22). Histologic sections were prepared and stained according to the manufacturer's instructions. As a negative control, the TdT enzyme was omitted from the assay. As a positive control, we used rat colon. Methyl-green and hematoxylin and eosin were used as counterstaining for noncardiac myocytes and colon cells, respectively. The number of TUNEL-positive cardiac myocyte nuclei was expressed as percentage of the total number of cardiac myocyte nuclei and called the apoptosis index. Six paraffin sections $(7 \mu \mathrm{m})$ in the coronal plane were obtained at $210-\mu \mathrm{m}$ distances from each tissue block. In each section, 10 random microscopic fields from the right and left ventricles were analyzed. TUNEL-positive cardiac myocytes were determined in 60 microscopic fields (light microscopy, 40× magnifications) from the right and left ventricles of each animal (12 rats in each study group; i.e., 4 rats at each time point). The reader was unaware of the disease stage.

\section{Picrosirius Red Staining}

To assess the total collagen in myocardial tissue, specimens were stained with picrosirius red F3BA (Sigma-Aldrich). Tissue sections (7- $\mu \mathrm{m}$ thickness) were treated and incubated in $0.1 \%$ picrosirius red. Before dehydration, the slides were treated with $0.01 \mathrm{~N}$ hydrochloride and mounted. Four animals per group were analyzed. Analysis was performed in 60 microscopic fields from the right and left ventricles, which were obtained as previously described (23). The picrosirius red-stained area was expressed as a percentage of the total ventricular tissue area using Image Pro Plus (version 5.02; Media Cybernetics). 


\section{Statistical Analysis}

Two-way ANOVA (factors treatment and time or disease stage) was performed. Because of a significant interaction between treatment and time, the initial analysis was followed by a 1-way analysis between treatments per time point or disease stage. For analysis of the echocardiographic data, these data were fit to an exponential curve (to reflect RV hypertrophy) through the mean values of RVFWT and RVEDD per time point. Delay in time was calculated as the average time between these best-fitted exponential curves, including all data points for which the treated groups had RVFWT and RVEDD above control values. Data are expressed as mean \pm SEM. A $P$ value of less than 0.05 was considered to indicate a statistically significant difference.

\section{RESULTS}

\section{Apoptosis Detection During Natural Time Course of RV Disease Progression}

Serial In Vivo ${ }^{99 m}$ Tc-Annexin Scintigraphy. Figure 1A shows ${ }^{99 \mathrm{~m}} \mathrm{Tc}$-annexin uptake in a monocrotaline rat in the RV hypertrophy stage and an age-matched control rat. Monocrotaline rats exhibited a biphasic time course of 99mTc-annexin uptake (Fig. 1B). Initially, ${ }^{99 m}$ Tc-annexin uptake increased and became significantly higher than baseline at $15 \mathrm{~d}$ after monocrotaline injection. Thereafter, ${ }^{99 m}$ Tc-annexin uptake declined toward the end of the study (RV failure) but remained elevated, compared with baseline. ${ }^{99 \mathrm{~m}} \mathrm{Tc}$-annexin uptake remained at baseline levels in control rats (Fig. 1B).

Ex Vivo Quantitative ${ }^{99 m}$ Tc-Annexin Autoradiography. Planar in vivo ${ }^{99 \mathrm{~m}} \mathrm{Tc}$-annexin scintigraphy did not allow us to distinguish whether the observed ${ }^{99 \mathrm{~m}}$ Tc-annexin uptake occurred in the right or left ventricle. To resolve this issue, we conducted ex vivo semiquantitative ${ }^{99 \mathrm{~m}} \mathrm{Tc}$-annexin autoradiography. Because the time course of in vivo ${ }^{99 \mathrm{~m}} \mathrm{Tc}-$ annexin uptake suggested differential ${ }^{99 \mathrm{~m} T c-a n n e x i n}$ uptake at the 3 successive RV disease stages, rats were sacrificed at each stage. We found increased ${ }^{99 \mathrm{~m}} \mathrm{Tc}$-annexin uptake in the right ventricle at all stages in monocrotaline rats, compared with control rats. This signal was highest at the RV hypertrophy stage (Fig. 2). In contrast, the autoradiography signal in the left ventricle did not change between disease stages in control rats and monocrotaline rats.

TUNEL Analysis. To obtain histologic confirmation that ${ }^{99 \mathrm{~m}}$ Tc-annexin uptake reflected apoptosis, we determined the apoptosis index using TUNEL assays of the right and left ventricles. Figure $3 \mathrm{~A}$ shows a TUNEL-positive cardiac myocyte in a monocrotaline rat heart and a TUNELpositive rat intestine cell (positive control). The stagedependent changes in apoptosis index of monocrotaline rats paralleled those of the ${ }^{99 \mathrm{~m}} \mathrm{Tc}$-annexin autoradiography signal, whereas the apoptosis index of control rats remained at baseline levels. Thus, the apoptosis index of monocrotaline rats was increased over age-matched control rats at all 3 stages and highest during RV hypertrophy. In LV, TUNEL-positive cardiac myocytes were virtually undetectable at all disease stages (data not shown).

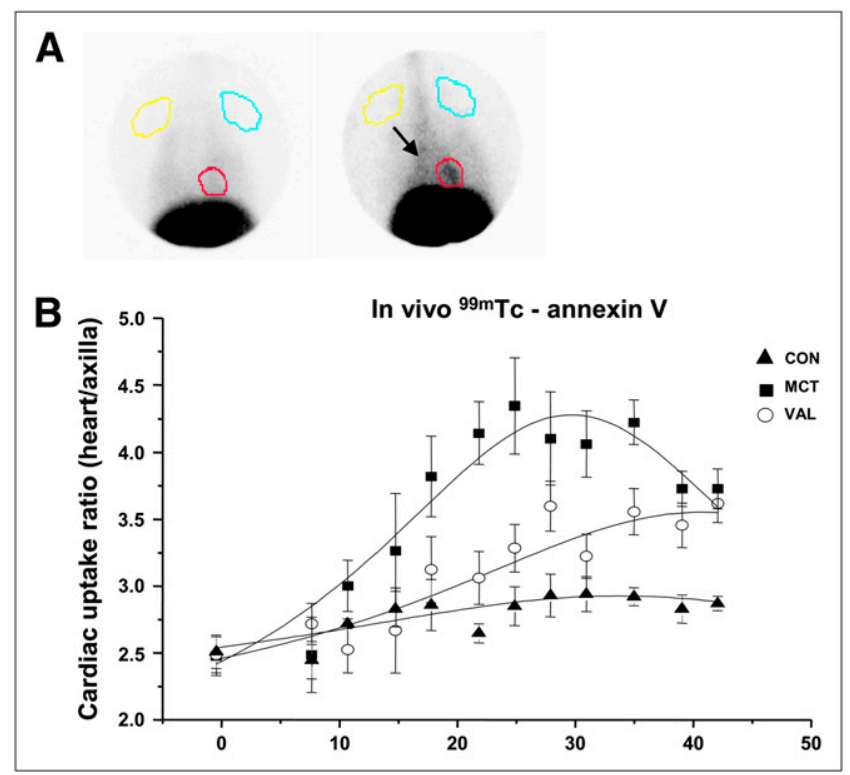

FIGURE 1. In vivo detection of apoptosis using 99mTcannexin scintigraphy. (A) Representative planar pinhole scintigrams $1 \mathrm{~h}$ after intravenous injection of ${ }^{99 \mathrm{~m} T c}$-annexin in monocrotaline rat in RV hypertrophy stage (right) and agematched control rat (left). Regions of interest were drawn around myocardium (red) and in extrathoracic soft tissue (yellow, blue) to determine cardiac uptake ratio. Note ${ }^{99 m T c-}$ annexin uptake in cardiac region and liver (arrow) of monocrotaline rat. (B) Cardiac uptake ratios at various times after monocrotaline injection ( $n=10$ per group). $P<0.05$ from day 15 until day 42 in monocrotaline rats and from day 18 until day 42 in valsartan rats vs. control rats. $\mathrm{CON}=$ agematched control; $\mathrm{MCT}=$ monocrotaline-injected rat; $\mathrm{VAL}=$ valsartan-treated rat. Data are mean \pm SEM.

Taken together, these findings indicate that serial ${ }^{99 \mathrm{~m}} \mathrm{Tc}-$ annexin scintigraphy allows for the noninvasive evaluation of the time course of RV apoptosis. To establish whether ${ }^{99 m}$ Tc-annexin scintigraphy may also be used to monitor the effects of therapies that counteract RV disease progression by reducing RV apoptosis, we next conducted ${ }^{99 \mathrm{~m}} \mathrm{Tc}$-annexin scintigraphy in valsartan rats.

\section{Apoptosis Detection During Long-Term Valsartan Treatment}

Serial Echocardiographic Measurements of $R V$ Function. The onsets of RV hypertrophy and RV dilation, as assessed by echocardiography, were delayed by $5.5 \pm 0.3 \mathrm{~d}$ and $4.4 \pm 0.3 \mathrm{~d}$, respectively, in valsartan rats, compared with monocrotaline rats (Figs. 4A and 4B).

In Vivo ${ }^{99 m}$ Tc-Annexin Uptake. In accordance with the delay in the onset of the successive RV disease stages in valsartan rats, the increase of in vivo ${ }^{99 \mathrm{~m}} \mathrm{Tc}$-annexin uptake of these rats was delayed, because ${ }^{99 \mathrm{~m}} \mathrm{Tc}$-annexin uptake became significantly higher than baseline only at $18 \mathrm{~d}$ after monocrotaline injection (Fig. 1B, circles). Moreover, the ${ }^{99 \mathrm{~m}} \mathrm{Tc}$-annexin uptake was lower in valsartan rats than in 
FIGURE 2. 99mTc-annexin autoradiography. (A) Representative short-axis myocardial slices from monocrotaline rat in RV hypertrophy stage (right) and age-matched control rat (left). Note higher signal intensity in right ventricle, compared with interventricular septum (IVS) or left ventricle (background). (B) Signal intensities of region of interest minus background (PSL/mm²), $n=10$ in control and monocrotaline groups each, with $n=3$ at RV hypertrophy and RV dilation, and $n=4$ in RV failure. ${ }^{\star} P<0.05$ vs. age-matched control rat. $\mathrm{CON}=$ age-matched control; MCT = monocrotaline-injected rat; $\mathrm{PSL}=$ photo-stimulated luminescence. Data are mean \pm SEM.

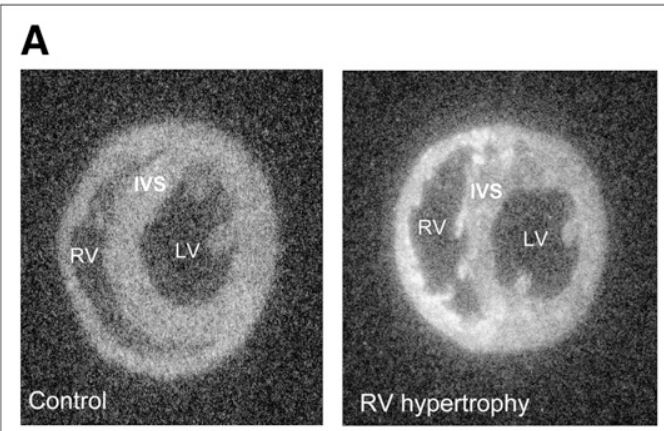

B

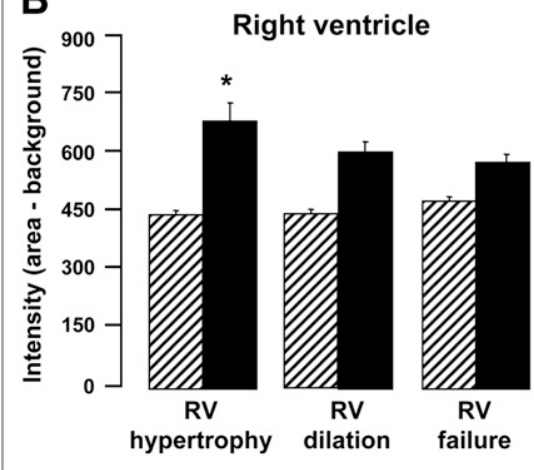

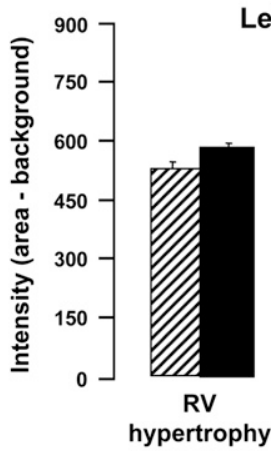

Left ventricle

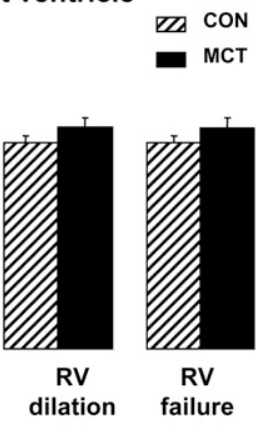

monocrotaline rats throughout disease progression. Still, the ${ }^{99 \mathrm{~m}} \mathrm{Tc}$-annexin uptake of valsartan rats was significantly increased over control rats at day 42 after monocrotaline injection.

TUNEL. The apoptosis index in valsartan rats exhibited the same time course as in monocrotaline rats (Fig. 3), but it was significantly lower in valsartan rats than in monocrotaline rats at the RV hypertrophy and RV dilation stages.

\section{Collagen Deposition}

Monocrotaline rats exhibited increased levels of interstitial collagen deposition. In contrast to the changes in RV apoptosis, which peaked at early RV disease stages, the amount of RV fibrosis continuously increased throughout $\mathrm{RV}$ disease progression, being highest during RV failure (Fig. 5A). In the left ventricle, collagen levels remained at baseline (not shown). Valsartan rats had a time course of $\mathrm{RV}$ fibrosis similar to monocrotaline rats, but RV fibrosis levels were significantly lower than in monocrotaline rats at all disease stages (Fig. 5B). LV fibrosis in valsartan rats remained unaltered, similar to that in monocrotaline rats (not shown).

\section{DISCUSSION}

We found increases in apoptosis during early stages of $\mathrm{RV}$ disease progression that can be monitored by serial in vivo ${ }^{99 \mathrm{~m} T c-a n n e x i n ~ s c i n t i g r a p h y . ~ M o r e o v e r, ~ d e l a y ~ i n ~}$ $\mathrm{RV}$ disease progression by valsartan treatment paralleled reduction in RV apoptosis, as detected using in vivo ${ }^{99 m}$ Tc-annexin scintigraphy. These findings demonstrate that noninvasive serial monitoring of myocardial apoptosis during RV disease progression is feasible and has potential clinical relevance.

We demonstrated that serial ${ }^{99 \mathrm{~m}} \mathrm{Tc}$-annexin scintigraphy can be used to monitor RV apoptosis throughout disease progression in a noninvasive manner. The ${ }^{99 \mathrm{~m}} \mathrm{Tc}$-annexin scintigram signal reflected apoptosis, as confirmed by 99mTc-annexin autoradiography and TUNEL, and exhibited a particular time course, peaking at early disease stages (hypertrophy) and declining thereafter (failure) but remaining significantly increased over baseline values at all RV disease stages. Furthermore, we found that a delay in RV disease progression by valsartan was attended by a reduction in RV apoptosis, as detected with serial in vivo ${ }^{99 \mathrm{~m}} \mathrm{Tc}-$ annexin scintigraphy. This observation not only illustrates that serial in vivo ${ }^{99 \mathrm{~m}} \mathrm{Tc}$-annexin scintigraphy may be used to monitor the effects of therapy aimed at counteracting apoptosis but also supports the notion that apoptosis is causally related to RV disease in this model. Still, this observation does not prove a causal relationship, because RV disease progression was not completely arrested and apoptosis was not completely abolished.

${ }^{99 \mathrm{~m}} \mathrm{Tc}$-annexin scintigraphy has been effectively used to noninvasively map regions of apoptosis in patients with various pathologies (24-28) and in an experimental model of autoimmune myocarditis (29). We anticipate that the ability to detect apoptosis serially in vivo using ${ }^{99 \mathrm{~m} T c-}$ annexin scintigraphy, as demonstrated in the present study, will not only facilitate mechanistic studies related to apoptosis but also allow for monitoring of the disease course or the response to various treatments aimed at counteracting $\mathrm{RV}$ failure. The use of serial echocardiography allowed us 


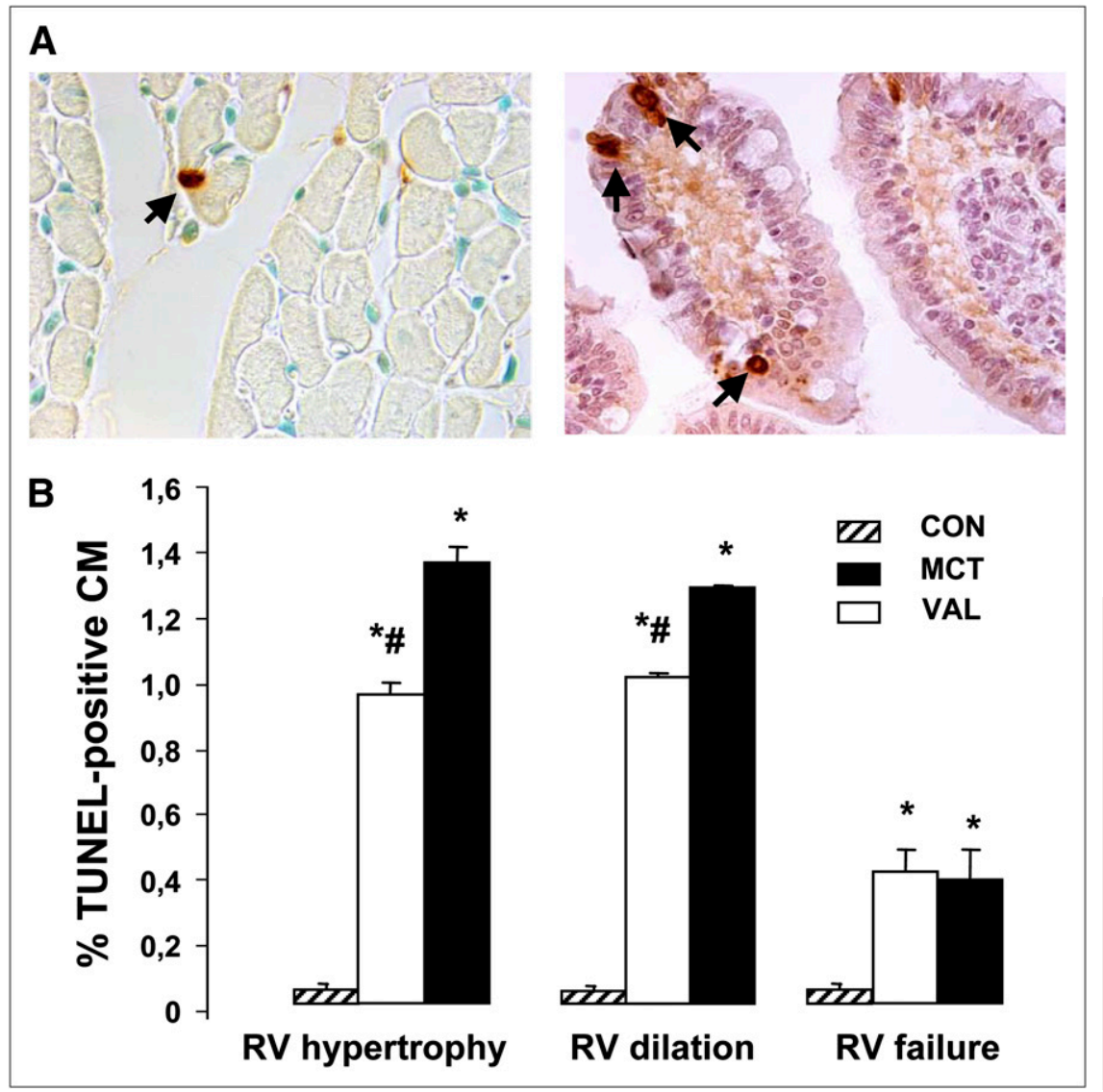

FIGURE 3. TUNEL staining. (A) Representative examples of apoptotic cell. TUNEL-positive cardiac myocyte (left; arrow), methyl green counterstain. Positive control, TUNEL-positive rat colon cell (right; arrows). (B) Apoptosis index during RV disease progression, $n=4$ per disease stage in each group. ${ }^{\star} P<$ 0.001 vs. control rat; $\# P<0.01$ vs. monocrotaline rat. $\mathrm{CM}=$ cardiac myocyte; $\mathrm{CON}=$ age-matched control; MCT = monocrotaline-injected rat; $\mathrm{VAL}=$ valsartan-treated rat. Data are mean \pm SEM.

to pinpoint apoptosis to specific RV disease stages. Defining the disease stages precisely is of great value in conducting such studies. The occurrence of apoptosis during early RV disease stages suggests a potentially beneficial effect of apoptosis inhibition. We found that apoptosis started early and declined when clinically manifest RV failure occurred. This finding is consistent with other pressure-overload mediated RV disease studies $(11,12)$.

Our findings that valsartan administration delayed RV disease progression correspond to the reported protective effects of olmesartan in the same animal model (14). Yet, they contrast with studies that showed that losartan did not prevent RV hypertrophy in the monocrotaline model $(30,31)$. These discrepancies may be due to greater potency of newer ARBs (valsartan, olmesartan) over first-generation ARBs (losartan). Furthermore, our results are consistent with the Valsartan Heart Failure Trial, which showed that valsartan attenuates morbidity in LV failure patients $(32,33)$.

Chronic activation of the renin-angiotensin-aldosterone system plays an important role in the structural and functional pathogenesis of heart failure. Moreover, it is now generally accepted that apoptosis is mediated via type 1 angiotensin II receptors (AT1) $(15,16)$. Leri et al. (34) and $\mathrm{Li}$ et al. (35) demonstrated in vitro that myocyte stretch-induced apoptosis can be inhibited by losartan.

Our study shows that delay in RV disease progression is not only associated with a reduction in apoptosis but also associated with reduced collagen deposition. The mechanisms leading to angiotensin II-induced fibrosis are thought to be at least in part mediated through growth factor pathways induced by AT1 activation (36,37). Extracellular
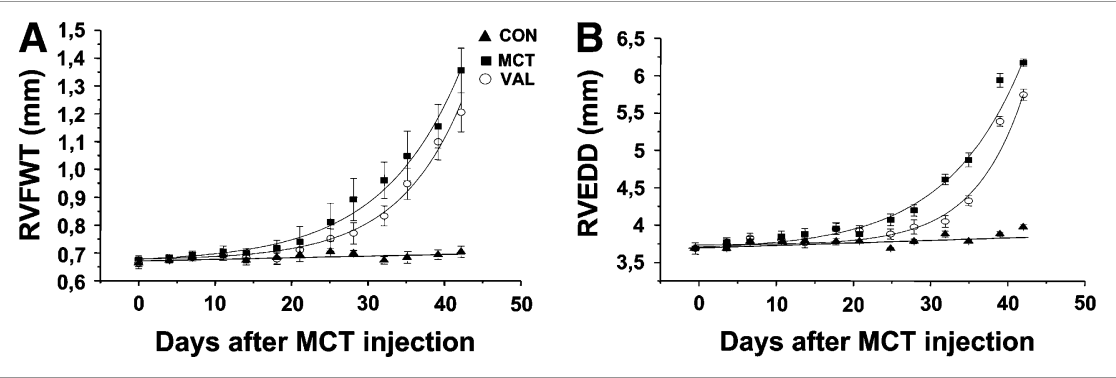

FIGURE 4. Time courses of development of RV hypertrophy and RV dilation. (A) Time course of development of RV hypertrophy assessed echocardiographically by RVFWT. (B) Time course of development of RV dilation assessed echocardiographically by RVEDD. CON = age-matched control; MCT = monocrotaline-injected rat; VAL = valsartan-treated rat. Data are mean \pm SEM. 


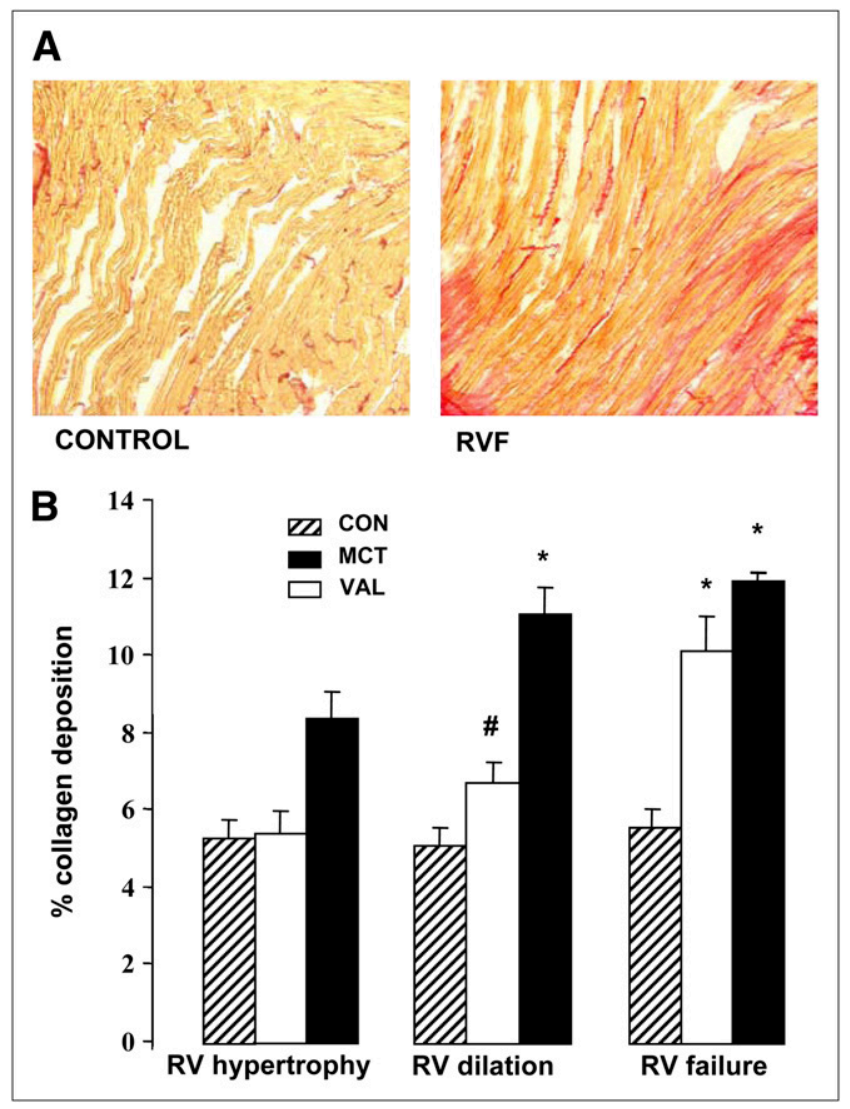

FIGURE 5. Collagen deposition. (A) Representative sections of interstitial fibrosis using picrosirius red staining from control rat (left) and monocrotaline rat with RV failure (RVF; right). Note homogeneous distribution of picrosirius red staining in right ventricle during RV failure. (B) Quantification of collagen deposition during $\mathrm{RV}$ disease progression, $n=4$ per group; ${ }^{\star} P<0.01$ vs. age-matched controls. $\mathrm{CON}=$ agematched control; $\mathrm{MCT}=$ monocrotaline-injected rat; $\mathrm{VAL}=$ valsartan-treated rat. Data are mean \pm SEM.

matrix accumulation in the cardiovascular system is involved in vascular and cardiac hypertrophy and heart failure. Thus, reduction in these changes may contribute to the beneficial effects of chronic AT1 receptor blockade on development of RV failure.

Although our findings are in line with previous studies in LV disease that provided clear evidence that apoptosis promotes heart failure, estimates regarding the amount of cell death from apoptosis, and the role of apoptosis in the progression into heart failure, are still controversial. The reported percentage of apoptosis in end-stage LV failure is similar to our findings in the RV failure stage (38). However, the contribution of apoptosis to disease progression remains controversial. Studies from a transgenic mouse model of cardiac-restricted expression of ligandactivated pro-caspase 8 have demonstrated that even low levels of cardiac myocyte apoptosis are sufficient to cause lethal dilated cardiomyopathy (39). Furthermore, in endstage LV failure, oncosis and autophagic cell death have also been reported to play an important role in disease progression (40). The role of these alternative modes of cell death in RV disease progression remains to be explored.

Our study has several limitations. Recent studies have shown that phosphatidylserine externalization occurs not only in apoptosis but also in activated macrophages. Furthermore, it has been shown that annexin binds to externalized phosphatidylserine and can also be internalized through an annexin-specific mechanism. These findings suggest that annexin cannot exclusively be used as a marker of apoptosis but can also be used to visualize inflammation (41).

In our study, we observed a similar pattern in ${ }^{99 \mathrm{~m}} \mathrm{Tc}-$ annexin and TUNEL staining throughout the time course of the development of RV failure. However, because double staining was not performed, we cannot exclude the possibility that noncardiac myocytes were visualized with 99mTc-annexin. Despite these limitations, we have shown that the stage-dependent changes in the apoptosis index of monocrotaline rats paralleled those of the ${ }^{99 \mathrm{~m}} \mathrm{Tc}$-annexin scintigraphy, which we believe is strongly associated with apoptotic cardiac myocytes in our animal model.

\section{CONCLUSION}

In vivo ${ }^{99} \mathrm{~m}$ Tc-annexin scintigraphy may be used for the serial assessment of apoptosis throughout RV disease progression and the efficacy of treatments aimed at counteracting apoptosis. Thus, in vivo ${ }^{99 \mathrm{~m}} \mathrm{Tc}$-annexin scintigraphy is a valuable tool for mechanistic studies-drug development and clinical management alike-because of its easy implementation and its noninvasive nature.

\section{ACKNOWLEDGMENTS}

We are grateful to Dr. Alexandre T. Soufan for his expertise in developing the software analysis. This work was supported in part by the Royal Netherlands Academy of Arts and Sciences (KNAW) and Netherlands Organization for Scientific Research (NWO, ZonMW VICI 918.86.616).

\section{REFERENCES}

1. Haddad F, Doyle R, Murphy DJ, Hunt SA. Right ventricular function in cardiovascular disease, part II: pathophysiology, clinical importance, and management of right ventricular failure. Circulation. 2008;117:1717-1731.

2. Voelkel NF, Quaife RA, Leinwand LA, et al. Right ventricular function and failure: report of a National Heart, Lung, and Blood Institute working group on cellular and molecular mechanisms of right heart failure. Circulation. 2006;114: 1883-1891.

3. Bleasdale RA, Frenneaux MP. Prognostic importance of right ventricular dysfunction. Heart. 2002;88:323-324.

4. Abbate A, Scarpa S, Santini D, et al. Myocardial expression of survivin, an apoptosis inhibitor, in aging and heart failure: an experimental study in the spontaneously hypertensive rat. Int J Cardiol. 2005;111:371-376.

5. Feldman AM, Weinberg EO, Ray PE, Lorell BH. Selective changes in cardiac gene expression during compensated hypertrophy and the transition to cardiac decompensation in rats with chronic aortic banding. Circ Res. 1993;73:184-192.

6. Berenji K, Drazner MH, Rothermel A, Hill JA. Does load-induced ventricular hypertrophy progress to systolic heart failure? Am J Physiol Heart Circ Physiol. 2005;289:H8-H16. 
7. Anversa P, Leri A, Beltrami CA, Guerra S, Kajstura J. Myocyte death and growth in the failing heart. Lab Invest. 1998;78:767-786.

8. Weber KT. Targeting pathophysiological remodeling: concepts of cardioprotection and reparation. Circulation. 2000;102:1342-1345.

9. Sabbah HN, Stein PD, Kono T, et al. A canine model of chronic heart failure produced by multiple sequential coronary microembolizations. Am J Physiol. 1991;260:H1379-H1384.

10. Schaper J, Froede R, Hein S, et al. Impairment of the myocardial ultrastructure and changes of the cytoskeleton in dilated cardiomyopathy. Circulation. 1991; 83:504-514.

11. Ecarnot-Laubriet A, Assem M, Poirson-Bichat F, et al. Stage-dependent activation of cell cycle and apoptosis mechanisms in the right ventricle by pressure overload. Biochim Biophys Acta. 2002;1586:233-242.

12. Ikeda S, Hamada M, Hiwada K. Cardiomyocyte apoptosis with enhanced expression of P53 and Bax in right ventricle after pulmonary arterial banding. Life Sci. 1999;65:925-933.

13. Blankenberg FG, Katsikis PD, Tait JF, et al. In vivo detection and imaging of phosphatidylserine expression during programmed cell death. Proc Natl Acad Sci USA. 1998;95:6349-6354.

14. Kato T, Nasu T, Sonoda H, Ito KM, Ikeda M, Ito K. Evaluation of olmesartan medoxomil in the rat monocrotaline model of pulmonary hypertension. J Cardiovasc Pharmacol. 2008;51:18-23.

15. Cigola E, Kajstura J, Li B, Meggs LG, Anversa P. Angiotensin II activates programmed myocyte cell death in vitro. Exp Cell Res. 1997;231:363-371.

16. Li D, Tomson K, Yang B, Mehta P, Croker BP, Mehta JL. Modulation of constitutive nitric oxide synthase, bcl-2 and Fas expression in cultured human coronary endothelial cells exposed to anoxia-reoxygenation and angiotensin II: role of AT1 receptor activation. Cardiovasc Res. 1999;41: 109-115.

17. Guide for the Care and Use of Laboratory Animals. Washington, DC: National Academy Press; 1996.

18. Lalich JJ, Merkow L. Pulmonary arteritis produced in rats by feeding: Crotolaria spectabilis. Lab Invest. 1961;10:744-750.

19. Hardziyenka M, Campian ME, de Bruin-Bon RHACM, Michel MC, Tan HL. Sequence of echocardiographic changes during development of right ventricular failure in rat. J Am Soc Echocardiogr. 2006;19:1272-1279.

20. Habraken JBA, de Bruin K, Shehata M, et al. Evaluation of high-resolution pinhole SPECT using a small rotating animal. J Nucl Med. 2001;42:1863-1869.

21. Knol RJJ, de Bruin K de Jong J, van Eck-Smit BLF, Booij J. In vitro and ex vivo storage phosphor imaging of short-living radioisotopes. J Neurosci Methods. 2008; 168:341-357.

22. Gavrieli Y, Sherman Y, Ben-Sasson SA. Identification of programmed cell death in situ via specific labeling of nuclear DNA fragmentation. J Cell Biol. 1992;119:493-501.

23. Burke AP, Farb A, Tashko G, Virmani R. Arrhythmogenic right ventricular cardiomyopathy and fatty replacement of the right ventricular myocardium. Circulation. 1998;97:1571-1580.
24. Hofstra L, Liem IH, Dumont EA, et al. Visualisation of cell death in vivo in patients with acute myocardial infarction. Lancet. 2000;356:209-212.

25. Vriens P, Blankenberg F, Stoot J, et al. The use of technetium Tc $99 \mathrm{~m}$ annexin V for in vivo imaging of apoptosis during cardiac allograft rejection. $J$ Thorac Cardiovasc Surg. 1998;11:844-853.

26. Hofstra L, Dumont EA, Thimister PW, et al. In vivo detection of apoptosis in an intracardiac tumor. JAMA. 2001;285:1841-1842.

27. Kietselaer BL, Reutelingsperger CP, Boersma HH, et al. Noninvasive detection of programmed cell loss with ${ }^{99 \mathrm{~m}} \mathrm{Tc}$-labeled annexin A5 in heart failure. $\mathrm{J} \mathrm{Nucl}$ Med. 2007;48:562-567.

28. Narula J, Strauss HWPS. P.S.* I love you: implications of phosphatidyl serine (PS) reversal in acute ischemic syndromes. J Nucl Med. 2003;44:397-399.

29. Tokita N, Hasegawa S, Maruyama K, et al. ${ }^{99 \mathrm{~m}} \mathrm{Tc}-H y n i c-a n n e x i n ~ V$ imaging to evaluate inflammation and apoptosis in rats with autoimmune myocarditis. Eur $J$ Nucl Med Mol Imaging. 2003;30:232-238.

30. Cassis LA, Rippetoe PE, Soltis EE, Painter DJ, Fitz R, Gillespie MN. Angiotensin II and monocrotaline-induced pulmonary hypertension: effect of losartan (DuP 753), a nonpeptide angiotensin type 1 receptor antagonist. $J$ Pharmacol Exp Ther. 1992;262:1168-1172.

31. Kreutz R, Fernandez-Alfonso MS, Ganten D, Paul M. Effect of losartan on right ventricular hypertrophy and cardiac angiotensin I-converting enzyme activity in pulmonary hypertensive rats. Clin Exp Hypertens. 1996;18:101-111.

32. Cohn JN, Tagnoni G. Valsartan Heart Failure Trial Investigators. A randomized trial of the angiotensin-receptor blocker valsartan in chronic heart failure. $N$ Engl J Med. 2001;345:1667-1675.

33. Krum H, Casson P, Farsang C, et al. Effect of valsartan added to background ACE inhibitor therapy in patients with heart failure: results from Val-HeFT. Eur J Heart Fail. 2004;6:937-945.

34. Leri A, Liu Y, Claudio PP, et al. Insulin-like growth factor-1 induces Mdm2 and down-regulates p53, attenuating the myocyte renin-angiotensin system and stretch-mediated apoptosis. Am J Pathol. 1999;154:567-580.

35. Li D, Yang B, Phillip MI, Metha JL. Proapoptotic effects of ANG II in human coronary artery endothelial cells: role of AT1 receptor and PKC activation. Am J Physiol. 1999;276:H786-H792.

36. Pinto YM, Pinto-Sietsma EJ, Phillip T, et al. Reduction in left ventricular messenger RNA for transforming growth factor $\beta_{1}$ attenuates left ventricular fibrosis and improves survival without lowering blood pressure in the hypertensive TGR(mRen2)27 rat. Hypertension. 2000;36:747-754.

37. Sun Y, Ramires FJ, Zhou G, Ganjam VK, Weber KT. Fibrous tissue and angiotensin II. J Mol Cell Cardiol. 1997;29:2001-2012.

38. Olivetti G, Abbi R, Quaini F, et al. Apoptosis in the failing heart. $N$ Engl J Med. 1997;336:1131-1141.

39. Wencker D, Chandra M, Nguyen K, et al. A mechanistic role for cardiac myocyte apoptosis in heart failure. J Clin Invest. 2003;111:1497-1504.

40. Kostin S, Pool L, Elsässer A, et al. Myocytes die by multiple mechanisms in failing human hearts. Circ Res. 2003;92:715-724.

41. Laufer EM, Reutelingsperger CPM, Narula J, Hofstra L. Annexin A5: an imaging biomarker of cardiovascular risk. Basic Res Cardiol. 2008;103:95-104. 